\title{
Obituary
}

\section{John Edensor Littlewood}

Many readers will have learnt already of the death last September, at the age of 92, of Professor J. E. Littlewood, F.R.S., one of our Association's most distinguished members.

Other journals will be carrying detailed accounts of Littlewood's outstanding contributions to research in pure mathematics. It may therefore suffice here to re-quote (from a review by J. C. Burkill in Gazette No. 323, February 1954) the assessment by the President of the Royal Society at the presentation to him of the Sylvester medal in 1943: "Into the theory of numbers, that subject where problems are so easy to state but so hard to prove, an entirely new spirit was infused when the methods of analysis were brought to bear upon it. The credit for attaining the highest results, for perfecting the methods, and for inspiring an enthusiastic following among so many of the ablest mathematicians at home and abroad, all belongs to Hardy and to Littlewood. ... Littlewood, on Hardy's own estimate, is the finest mathematician he has ever known. He was the man most likely to storm and smash a really deep and formidable problem; there was no one else who could command such a combination of insight, technique and power."

But it seems likely that, in electing him to Honorary Membership in 1937, the Council of the Mathematical Association had also other qualities in mind. For Littlewood had an abiding concern for the teaching and communicating of mathematics, and had in his own writing the gift of "getting the message across" and avoiding the obfuscation of trivial detail. In the review already referred to, Burkill wrote: "The author has been described as 'a legendary rather than a public figure' (Nature, Vol. 165, p. 341) and it is likely that he is known by sight to few members of the Mathematical Association except his former pupils." Nevertheless, there must be many of us who feel we knew him not so much through his Theory of functions as through $A$ mathematician's miscellany-surely the best of all mathematical bedside books. Here, in a blazingly honest account of his own mathematical education, in a set of notes on ballistics taken from his experiences in the 1914-1918 war, in a deeply thoughtful assessment of the place of Ramanujan in the history of mathematics, in a super-witty paper on "Large numbers"--these last two reprinted from Gazettes (No. 200, April 1929 and No. 300, July 1948 respectively)-we meet a man of broad mathematical sympathies, strongly held opinions which he is not afraid to express, and an immense sense of mathematical fun.

On hearing of Littlewood's death, one of our Vice-Presidents, Mr H. V. Lowry, sent me the following reminiscence: "I was a lucky student who attended his lectures in 1919, and was not forced to leave. There were about three times as many students as the room would hold, so at the first 
lecture he cut the numbers down by saying: 'Are there any here who have not read more than the first chapter of Hardy's book? Well, you can go as I shall be starting beyond that.... Are there any who have read more than the first five chapters? Well, you can go too.' I enjoyed the lectures very much."

"Enjoy" seems to be an appropriate word on which to end this quite inadequate tribute to a great man. For many members of our Association Littlewood was the personification of Cambridge mathematics at one of its peaks; it has been a privilege for us to occupy a tiny portion of his shadow.

D.A.Q.

\section{Notes}

\subsection{Golden weaves}

In Mathematical snapshots ([1], p. 49) Steinhaus points out that a surprisingly uniform division of a line-segment can be produced by using the fractional portion of integral multiples of the golden ratio $\phi=\frac{1}{2}(\sqrt{ } 5+1) \approx$ 1.6180 , giving a sequence $0.6180 \ldots, 0.2361 \ldots, 0.8541 \ldots$, etc. In performing sequential point sampling, this property has been used ([2], p. 32$)$ in optimising the search for a single maximum or minimum. While attempting to extend this sequential division from a line to that of an area, a remarkable set of weave patterns was discovered. These weaves, when formed on a square loom, appear to be infinite, and as each new thread of the weave is drawn, it will divide in the golden ratio any previously formed line-segment that it crosses; for this reason these are called "golden weaves".

To illustrate, let us assume the weave is started at the lower left corner of a square loom. The first thread is drawn such that the angle it makes with the base of the loom has a tangent equal to $(n+\phi) /(n+\phi+1)$, where $n$ is a non-negative integer (Figs. 1 and 2). Each time a thread or line meets the side of the square, it is reflected in the same manner as a ray of light to form the next line of the weave. Following the third reflection it can be seen that the first cross-over occurs, and thereafter each new line will usually cross several previously formed lines. For $n=0$, the first cross-over (Fig. 1a) divides the first line into segments whose ratio is golden. Then as additional cross-overs occur any previously formed line-segments are successively divided into new segments whose ratio is also golden (Figs. $1 \mathrm{~b}$ and $1 \mathrm{c}$ ). For weaves when $n$ is greater than zero it is observed that the first cross-over occurs after the third reflection, but golden division does not begin until after $2 n+3$ reflections (Fig. 2).

We shall prove this for the first two intersections in the case $n=0$. In Figs. 1a and $1 \mathrm{~b}$ properties of parallelograms and isosceles triangles ensure that all the lengths marked $a$ are equal, and similarly all the lengths marked 